\title{
Insight into Resonant Activation in Discrete Systems
}

\author{
O. Flomenbom and J. Klafter \\ School of Chemistry, \\ Raymond 85 Beverly Sackler Faculty Of Exact Sciences, \\ Tel-Aviv University, Tel-Aviv 69978, Israe*
}

(Dated: 21st November 2018)

\begin{abstract}
The resonant activation phenomenon (RAP) in a discrete system is studied using the master equation formalism. We show that the RAP corresponds to a non-monotonic behavior of the frequency dependent first passage time probability density function $(p d f)$. An analytical expression for the resonant frequency is introduced, which, together with numerical results, helps understand the RAP behavior in the space spanned by the transition rates for the case of reflecting and absorbing boundary conditions. The limited range of system parameters for which the RAP occurs is discussed. We show that a minimum and a maximum in the mean first passage time (MFPT) can be obtained when both boundaries are absorbing. Relationships to some biological systems are suggested.
\end{abstract}

PACS number: 05.40.Ca, 82.20.Db, 02.50.Cw

\section{INTRODUCTION}

Noise induced escape of a particle from a potential well has been a fundamental way for describing various processes in biology, chemistry, and physics, since the seminal work of Kramers [1]. More recently, it has been suggested that for some systems the potential itself fluctuates in time. A few examples are: the transport of ions and bio-polymers through membrane channels [2]-[4], enzymatic kinetics 5 , and the rebinding of ligand-protein complexes [6, 7]. The basic formulation of noise induced escape from a fluctuating environment is obtained by making the potential term of the stochastic differential equation (e.g. a white noise overdamped Langevin equation) change with a frequency $\gamma$ between two states. Doering and Gadoua showed that for a fluctuating system, the MFPT, $\tau$, from a reflecting boundary to an absorbing boundary, may show a minimum as a function of $\gamma[8]$. The occurrence of a minimum in $\tau(\gamma)$ was termed the resonant activation phenomenon (RAP). This has been followed by an extensive theoretical work to understand the nature of the RAP [9]- 16 , along with experimental efforts to find systems displaying RAP [2, 17]. The theoretical works have been mainly focused on checking the effect of different potentials on the RAP.

In this paper we study the discrete case RAP using coupled master equations (ME). We show that the RAP is only one of the properties that stem from the nonmonotonic behavior of the frequency dependent first passage times $(\mathrm{FPT}) p d f, F_{\gamma}(t)$, and which are related to frequency dependent minima in the first and higher moments of $F_{\gamma}(t)$. We introduce an analytical expression for the dependence of the frequency that minimizes $\tau(\gamma)$ on the system transitions rates. We show that the RAP is obtained only when certain conditions imposed on the transition rates are fulfilled. Analyzing these conditions we come up with an instructive understanding regarding the nature of the RAP. In addition, a novel behavior of the MFPT is obtained when changing the reflecting boundary into an absorbing one: the coexistence of a minimum and a maximum in $\tau(\gamma)$.

\section{THEORETICAL FRAMEWORK}

We describe the escape from a system that fluctuates between two configurations $A$ and $B$, using the coupled ME:

$$
\frac{\partial}{\partial t}\left(\begin{array}{c}
\vec{P}_{A}(t) \\
\vec{P}_{B}(t)
\end{array}\right)=\left(\begin{array}{cc}
\mathbf{A}-\mathbf{I} \gamma & \mathbf{I} \gamma \\
\mathbf{I} \gamma & \mathbf{B}-\mathbf{I} \gamma
\end{array}\right)\left(\begin{array}{c}
\vec{P}_{A}(t) \\
\vec{P}_{B}(t)
\end{array}\right) .
$$

$\vec{P}_{A}(t)\left(\vec{P}_{B}(t)\right)$ is an $n$-dimensional column vector, whose $j$ element is the $p d f$ to occupy site $j$ of the $A(B)$ configuration at time $t$. The transition between each site $j$ in one configuration and its counterpart in the second configuration occurs with a flipping frequency $\gamma$, see Fig. 1. $\mathbf{I}$ is the unit matrix of $n$ dimensions introduced in Eq. (1) to indicate the configurational coupling. Movement along each of the configurations, $A$ and $B$, is governed by the square $n$-dimensional tridiagonal propagation matrices $\mathbf{A}$ and $\mathbf{B}$, respectively, whose elements are the transition rates (Fig. 1). The choice of the matrices $\mathbf{A}$ and $\mathbf{B}$ corresponds to an equivalent choice of potential profiles and boundary conditions in the continuum case. In what follows we set a reflecting boundary at site $j=n$, and an

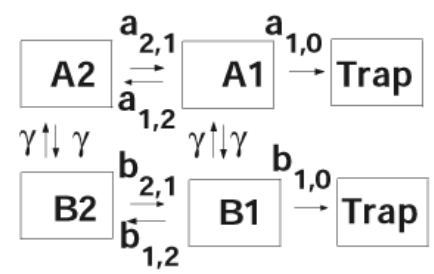

Figure 1: Schematic illustration of the exit problem in a fluctuating environment for $n=2$. For an invariant system $a_{1,0}=a_{2,1}=a_{f}, a_{1,2}=a_{b}$, and $b_{1,0}=b_{2,1}=b_{f}, b_{1,2}=b_{b}$. For the particular invariant birth-death system $a_{b}=0$, and $b_{f}=0$. 
absorbing boundary, as a trap, at site $j=0$, unless otherwise indicated. Fig. 1 shows a schematic illustration of the coupled system for $n=2$.

The FPT $p d f$ is defined by $F_{\gamma}(t)=\partial\left(1-S_{\gamma}(t)\right) / \partial t$, where $S_{\gamma}(t)$ is the survival probability; namely, the probability of not reaching the site $j=0$ until time $t . S_{\gamma}(t)$ is obtained by summing the elements of the vector that solves Eq. (1), $S_{\gamma}(t)=\vec{U}_{2 n} \mathbf{E} e^{\mathbf{D} t} \mathbf{E}^{-1} \vec{P}_{2 n}(0)$. Here $\vec{U}_{2 n}$ is the summation row vector of $2 n$ dimensions, $\vec{P}_{2 n}(0)$ is the initial condition column vector, $\left[\vec{P}_{2 n}(0)\right]_{j}=$ $\left(\delta_{x, j} P_{A, 0}+\delta_{x+n, j} P_{B, 0}\right)$, where $x$ is the initial site, and the process starts in the $A(B)$ configuration with probability $P_{A, 0}\left(P_{B, 0}\right)$. Unless otherwise specified, we use $x=n$ as a starting site, and $P_{A, 0}=P_{B, 0}=1 / 2$, as suggested from the single configurational flipping frequency. The definite negative real part eigenvalues matrix $\mathbf{D}$ is obtained through the similarity transformation: $\mathbf{D}=\mathbf{E}^{-1} \mathbf{H E}$, where $\mathbf{H}$ is the matrix given on the right hand side of Eq. (1), and $\mathbf{E}$ and $\mathbf{E}^{-1}$ are the eigenvectors matrix, and its inverse, of $\mathbf{H}$.

\section{RESULTS AND DISCUSSION}

We start by computing $F_{\gamma}(t)$ for an invariant birthdeath system. By an invariant system we mean that the transition rates are independent of the site index, $j$; namely, $m_{j, j-/+1}=m_{f / b}$ for configuration $M$, where $m_{f / b}$ represents $a_{f / b}$ and $b_{f / b}$ transition rates, and $M$ stands for $A$ and $B$. A birth-death system means that the movement in each configuration occurs only in one direction, i.e. $a_{b}=b_{f}=0$. Clearly, the term birth-death indicates that the particle [when simulating Eq. (1)] can move only towards its "death" (the trap) when it is subjected to the dynamics of the $A$ configuration, and in this sense, when flipping to the $B$ configuration occurs it is "born" (or "resurrected"). Therefore, for the birth-death system, the fluctuations are between a configuration which acts as a "barrier", the birth configuration, and a configuration acting as a "valley", the death configuration. Note that a single-rate (namely, $a_{f}=b_{b}$ ) invariant birthdeath system is similar to the system studied by Doering and Gadoua 8], where the derivatives of the two linear potentials are sign opposite and equal in the absolute values.

Fig. 2 shows $F_{\gamma}(t)$ for a single-rate invariant birthdeath system and $n=10$. At short to intermediate times, $F_{\gamma}(t)$ displays a peak that shifts towards larger times with $\gamma$. This peak represents the exiting of the initial population of configuration $A, \delta_{x, j} P_{A, 0}$, for small $\gamma$, and the overall initial condition for large $\gamma$. At longer times and intermediate $\gamma$, a minimum in $F_{\gamma}(t)$ appears as a function of $\gamma$ that represents the fastest exit mainly of the initial $B$ population. Accordingly, the minimum in the MFPT $\tau(\gamma)$ is a consequence of the shape of $F_{\gamma}(t)$, and is therefore reflected in higher moments of $F_{\gamma}(t)$ as well.

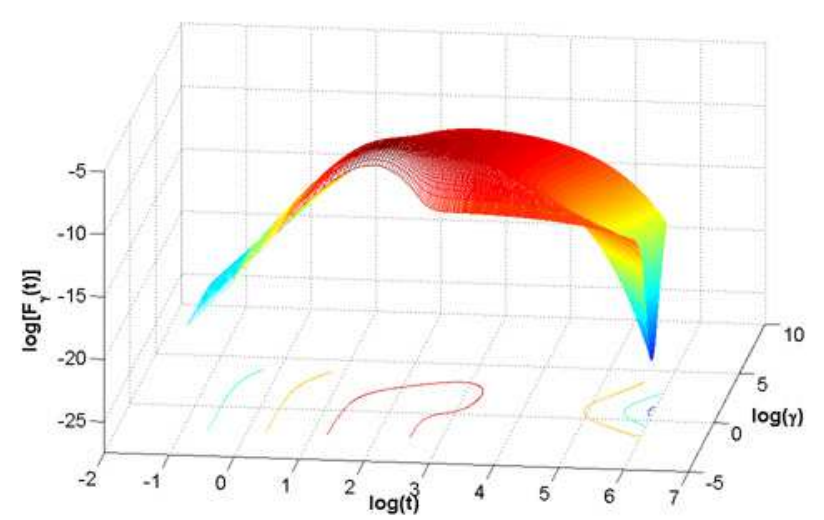

Figure 2: $F_{\gamma}(t)$ as a function of $t$ and $\gamma$ on a log-log-log plot (natural $\log$ ), for a single rate invariant birth-death system with $n=10$, and $a_{f}=b_{b}=1$. Also shown is $F_{\gamma}(t)$ projections.

To study the RAP we start by computing the MFPT. In general, the $s^{t h}$ moment of $F(t)$ is obtained by inverting matrix $\mathbf{H}: \tau^{s}=\int_{0}^{\infty} t^{s} F(t) d t=$ $s ! \vec{U}_{2 n}(-\mathbf{H})^{-s} \vec{P}_{2 n}(0)$. Using the projection operator techniques for $\mathbf{H}^{-1}$ blocks [18], $\tau$ reads

$$
\begin{aligned}
\tau=\gamma \vec{U}_{n}\left(\mathbf{C}_{\mathbf{A}}+\mathbf{C}_{\mathbf{B}}\right) \vec{P}_{n}(0)- \\
-\vec{U}_{n}\left(\mathbf{C}_{\mathbf{A}} \mathbf{A}+\mathbf{C}_{\mathbf{B}} \mathbf{B}\right) \vec{P}_{n}(0) / 2,
\end{aligned}
$$

where $\mathbf{C}_{\mathbf{A}}=[\mathbf{A B}-\gamma(\mathbf{A}+\mathbf{B})]^{-1}, \mathbf{C}_{\mathbf{B}}=[\mathbf{B A}-\gamma(\mathbf{A}+\mathbf{B})]^{-1}$, and $\left[\vec{P}_{n}(0)\right]_{j}=\delta_{x, j}$. From Eq. (2) one can calculate $\tau(\gamma)$ for the limiting cases $\gamma \rightarrow 0$ and $\gamma \rightarrow \infty$. For $\gamma \rightarrow 0, \tau$ is the average of the MFPT of the uncoupled configurations, $A$ and $B, \tau=\left(\tau_{A}+\tau_{B}\right) / 2$, where $\tau_{M}=-\vec{U} \mathbf{M}^{-1} \vec{P}_{n}(0)$ is the MFPT of configuration $M$. For $\gamma \rightarrow \infty, \tau$ is the MFPT of an averaged fully coupled system, namely, $\tau=-\vec{U}_{n}\left(\frac{\mathbf{A}+\mathbf{B}}{2}\right)^{-1} \vec{P}_{n}(0)$. These are the expected limiting behaviors of the MFPT [8]-[12], [15, 16]. RAP is expected for intermediate flipping frequencies.

To obtain an analytical expression for the frequency that minimizes $\tau(\gamma), \gamma_{\min }$, we search for an extremum point (a minimum) of the function $\tau(\gamma)$, for an invariant system and $n=2$. We find $\gamma_{\min }$ to be a sum of two terms:

$$
\gamma_{\min }=\left[\gamma_{\min , 1} \geq 0\right]+\left[\gamma_{\min , 2} \geq 0\right],
$$

where the notations on the right hand side of Eq. 3 mean that each of the terms must be non negative to contribute to $\gamma_{\min }$, and

$$
\gamma_{m i n, 1}=\frac{a_{f}\left(3 b_{f}^{2}-a_{f} b_{b}\right)-b_{f}\left(3 a_{f}^{2}-b_{f} a_{b}\right)}{a_{f}\left(a_{f}-2 b_{b}\right)-b_{f}\left(b_{f}-2 a_{b}\right)},
$$

and

$$
\gamma_{m i n, 2}=\frac{a_{f}\left(b_{f}^{2}-a_{f} b_{b}\right)-b_{f}\left(a_{f}^{2}-b_{f} a_{b}\right)}{a_{f}\left(a_{f}+2 b_{b}\right)-b_{f}\left(b_{f}+2 a_{b}\right)} .
$$


We note that the smallest system that exhibits the RAP requires a three site system, which is a specific case of the system shown in Fig. 1, with, for example, $b_{1,2}=$ $b_{2,1} \rightarrow \infty$. However, in what follows we consider systems with finite transition rates.

For the birth-death system Eq. (3) reduces to:

$$
\gamma_{\min }=\frac{a_{f}}{2-a_{f} / b_{b}} .
$$

The simple form of Eq. (6) provides an insight into the nature of the RAP. It immediately implies the requirement $a_{f} / b_{b}<2$ for RAP to occur. For $b_{b} \gg a_{f}$, $\gamma_{\min }=\left(\tau_{A}\right)^{-1}$, where $\tau_{A}$ is the first moment, $s=1$, of $F(t)$ for a death system, $\tau_{A}^{s}=(n)_{s} / a_{f}^{s}$, where $(n)_{s}=$ $(n+s-1) ! /(n-1) !$. This optimal frequency means that $\delta_{x, j} P_{A, 0}$ has exited the interval, on average, while the first configurational transition occurred, and the same holds for $\delta_{x, j} P_{B, 0}$, for the second configurational transition. Because the probability (particles) can exit the interval only when it is subject to the $A$ configuration dynamics, a situation where the $A$ configuration is empty but not the $B$ configuration, means a "waste" of time with regards to fastest interval exiting. This is the case for $\gamma<\gamma_{\min }$. For $\gamma>\gamma_{\text {min }}$, not all $\delta_{x, j} P_{A, 0}$ exited the interval, while the first configurational transition occurred, meaning that another cycle of flipping is required to exit the system. This leads again to a "waste" of time with regards of fastest interval exiting. At $\gamma=\left(\tau_{A}\right)^{-1}$, only one configurational change occurs, and costs the minimal time for exiting the interval.

The special feature that $a_{f} / b_{b}<2$ is needed for RAP suggests that the rate along the birth configuration must be, at least, as fast as those along the death configuration for the RAP to be obtained. For an invariant birthdeath system to show the RAP, the ratio $a_{f} / b_{b}$ must fulfil $a_{f} / b_{b} \leq 3$ asymptotically, which is demonstrated in Fig. 3. Note that Fig. 3 spans both degrees of freedom of the invariant birth-death case, the size $n$, and the ratio $a_{f} / b_{b}$. Scaling the time, $\widetilde{t}=t b_{b}$, leads to dimensionless rates $a_{f} / b_{b}$ and $\gamma / b_{b}$.

We emphasize that even for the simple invariant birthdeath system that fulfills the demand that the $B$ configuration rate is much larger than of configuration $A$ rates, the relation $\gamma_{\min }=\left(\tau_{A}\right)^{-1}$ might not be satisfied. To see that we calculate $\gamma_{\min }$ for a general birth-death system and $n=2$ (see Fig. 2):

$$
\gamma_{\text {min }}=\frac{\sqrt{a_{1,0} a_{2,1}}}{2-\sqrt{a_{1,0} a_{2,1}} / b_{1,2}},
$$

which for $\sqrt{a_{1,0} a_{2,1}} / b_{1,2} \rightarrow 0$, reduces to $\gamma_{\min }=$ $\sqrt{a_{1,0} a_{2,1}} / 2$, where $\left(\tau_{A}\right)^{-1}=a_{1,0} a_{2,1} /\left(a_{1,0}+a_{2,1}\right)$. Moreover, when $a_{1,0} \gg a_{2,1},\left(\tau_{A}\right)^{-1} \approx a_{2,1}, \gamma_{\text {min }}$ is unchanged, and is much larger than $\left(\tau_{A}\right)^{-1}$, implying that more than one configurational change occurs at the optimal flipping frequency. Reverse substitution $\gamma=\gamma_{\text {min }}$ and $\gamma=\left(\tau_{A}\right)^{-1}$ into $\tau(\gamma)$, results in $\tau\left(\gamma=\gamma_{\min }\right) \approx 2 / a_{2,1}$ and $\frac{\tau\left(\gamma=\gamma_{\min }\right)}{\tau\left(\gamma=\left(\tau_{A}\right)^{-1}\right)} \approx 0.8$. Note that for both values of $\gamma, \tau(\gamma)$ is independent of $b_{1,2}$ to be compared with $\tau(\gamma \rightarrow \infty) \approx \frac{b_{1,2}}{a_{1,0} a_{2,1}} . \quad \gamma_{\text {min }}$ has, therefore, a general functional form that not necessarily coincides with the MFPT of the faster configuration.

Going beyond the birth-death system, we first consider a case for which $\mathbf{B}=\lambda \mathbf{A}$. From Eqs.(3)-(5) we have, for $n=2, \gamma_{\min }<0$ for any positive $\lambda$. From numerical calculation we find that for $n>2$ there is no real positive $\gamma_{\min }$. Both the analytical and the numerical results imply that a system for which $\mathbf{A}$ and $\mathbf{B}$ commute does not exhibit the RAP.

The next case to be checked for the occurrence of the RAP is obtained by setting, $a_{f}=b_{b}=k$. Using $\widetilde{t}=k t$, the system transition rates are dimensionless, measured in units of $k$. This procedure leads to the reduced Eqs.(4$5)$ :

$$
\gamma_{\min , 1} / k=\frac{v^{2}[3+u]-3 v-1}{v[2 u-v]-1}
$$

and

$$
\gamma_{\min , 2} / k=\frac{v^{2}[1+u]-v-1}{3-v[2 u+v]}
$$

where $u=a_{b} / k$ and $v=b_{f} / k$. Fig. 4a shows $\gamma_{\min } / k$ as a function of $v$ for $u=1 / 2 . \gamma_{\min , 1} / k$ displays a maximum at $v_{\max }$, which is easily recovered from Eq. (8). For $v \leq v_{\max }, \gamma_{\min , 1} / k$ increases, which reflects the increase in the relative ability of the $B$ configuration to "help" the fastest exiting of configuration $A$. On the other hand, the decrease in $\gamma_{\min , 1} / k$ for $v \geq v_{\max }$, implies that the $B$ configuration movement towards the absorbing end be-

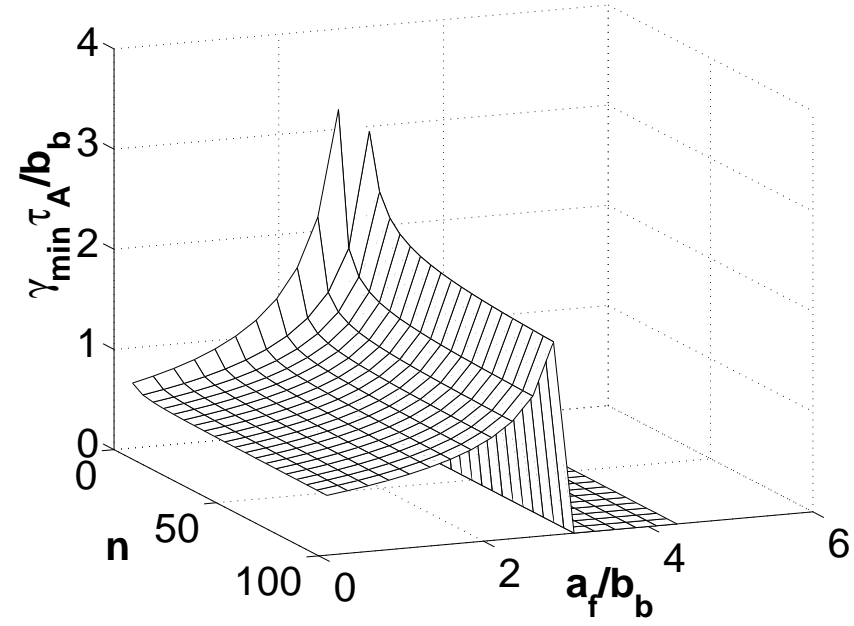

Figure 3: $\gamma_{\min } \tau_{A} / b_{b}$ as a function of $n$ and the ratio $a_{f} / b_{b}$. When $a_{f} / b_{b}>3$ the RAP does not exist, for any $n$. 


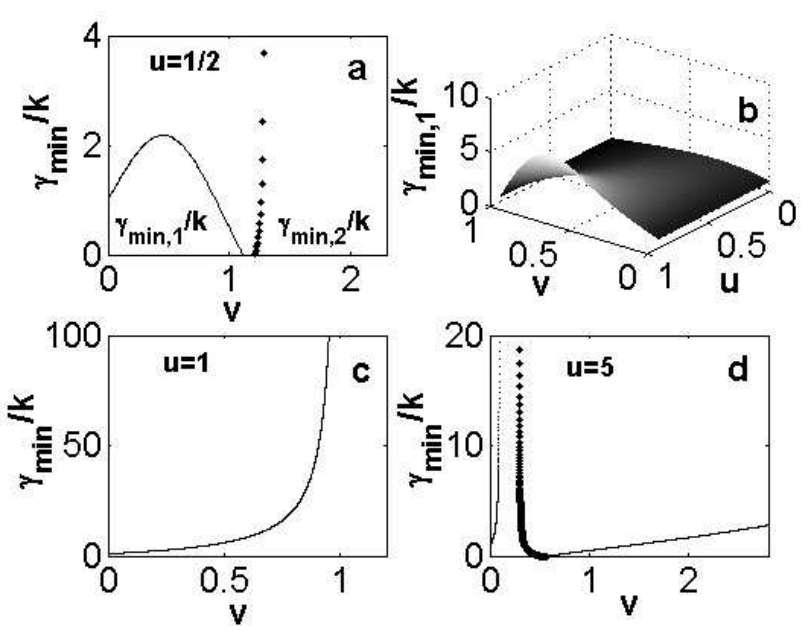

Figure 4: a: $\gamma_{\min } / k$ for $a_{f}=b_{b}=k$, and $u=1 / 2$, as a function of $v$. b: $\gamma_{\min , 1} / k$ as a function of $0 \leq u, v<1$. c: $\gamma_{\min } / k$ for $u=1$ as a function of $v$. d: $\gamma_{\min } / k$ for $u=5$ as a function of $v$.

comes fast enough "to stand on its own" for the accomplishment of this task. A resonant-free zone occurs in the region where $\tau_{A} \approx \tau_{B}$, and is followed by a short resonant region, where both configurations are trap oriented, namely, $u<1$ and $v>1$. Fig. $4 \mathrm{~b}$ shows that in the range $0 \leq u<1 \gamma_{\min , 1} / k$ is non-monotonic. Fig. 4c shows for $u=1$ the only non-zero $\gamma_{\min , 1} / k>0$ which diverges as $\frac{1}{1-v}$ when $v \rightarrow 1$, because for these system parameters $\mathbf{B}=\mathbf{A}$. For $u>1$ and $v \leq 1, \gamma_{\min } / k$ has two asymptotic lines, which define a resonant-free region. This happens when $\mathbf{B} \approx \lambda \mathbf{A}$. For $u \gg v>1, \gamma_{\min } / k \sim v / 2$. These two features are demonstrated in Fig. 4 d.

The above analysis is of importance since the coupled ME with $n=2$ can be used to model the kinetics of a conformationally changing enzyme. Such extended Michaelis-Menten models are appropriate for describing experiments performed on a single molecule level [5]. If we assume that there are two enzyme conformations, a specific stage of the enzymatic activity can be described by Fig. 1. From Eqs. (8) and (9), and more generally Eqs. (4) and (5), a relationship between the reaction rates and the conformational flipping frequency can be established for an optimal enzymatic activity. In addition, changes in the flipping rate value near the resonant frequency, which can be achieved for example by binding of other molecules to the enzyme, provide a simple and efficient mechanism for regulating the enzymatic activity, which is a well known issue in biology [19].

Finally, we study a system for which both ends are absorbing; namely, the reflecting boundary is replaced by an absorbing one, and the escape process starts at the middle site $(n=7$, and $x=4)$. The coupled invariant configurations are taken to have an opposite bias, i. e. the transition rates of the $B$ configuration are $b_{f}=0.175$, $b_{b}=1$, which give rise to a left side bias as defined in Fig.
1 , whereas in the $A$ configuration $a_{f}=3.5$, and $a_{b}<a_{f}$ give rise to a right side bias. A global minimum and a global maximum in $\tau(\gamma) / \tau(\gamma \rightarrow \infty)$ can occur (Fig. 5b). The minimum and maximum appear at the neighborhood of the points $\gamma_{\min } \tau_{B}=1$, and $\gamma_{\max } \tau_{A}=1$, respectively. This behavior is sensitive to the value of $a_{b}$ (Fig. 5a). For $a_{b} \rightarrow 0$ the global extremum points reduce to local extremum points. When $a_{b} \rightarrow a_{f}, \tau(\gamma) / \tau(\gamma \rightarrow \infty)$ is a monotonically increasing function of $\gamma \tau_{A}$.

We note that these boundary conditions for a fluctuating system have been used to describe the translocation of a single stranded DNA through a conformationally changing nanopore [4]. For this case no resonance occurred because of the physical conditions that imposed the relation $\mathbf{B}=\lambda \mathbf{A}$. However, for systems that are described by matrices $\mathbf{A}$ and $\mathbf{B}$ that do not commute and for two absorbing ends, a change in $\gamma$ in the vicinity of the extremal points, leads to a drastic change in the average time during which the system is occupied, and, therefore, emphasizes the importance of the frequency of fluctuation as a control parameter.

\section{CONCLUSIONS}

To conclude, in this paper we revisited the resonant activation phenomenon. We studied the origin of the RAP and the requirements under which this phenomenon can be observed. We showed that for a single rate invariant birth-death system the RAP is a consequence of a general phenomenon, which is a non-monotonic behavior of $F_{\gamma}(t)$ along the frequency axis for large time. We characterized the conditions for which an invariant birth-death system exhibits the RAP, and broaden these conditions by examining more general systems. Relationship between the RAP and biological activity was suggested. In addition, we introduced a new property of the MFPT, the coexistence of a minimum and a maximum in the flipping frequency dependent MFPT, $\tau(\gamma)$.
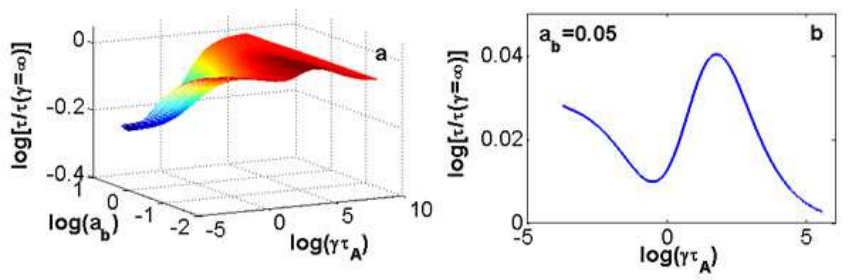

Figure 5: a: A log-log-log plot (natural $\log$ ) of $\tau(\gamma) / \tau(\gamma \rightarrow$ $\infty)$ as a function of $a_{b}$ and $\gamma \tau_{A}$, for two absorbing ends in an invariant system, with $a_{f}=3.5, b_{f}=0.175, b_{b}=1, n=7$, and $x=4$. b: Profile of the left figure for $a_{b}=0.25$ exposes a global minimum and a global maximum in $\tau(\gamma) / \tau(\gamma \rightarrow \infty)$. 
* We thank Attila Szabo for fruitful discussions.

[1] H. A. Kramers, Physica 7, 284 (1940).

[2] M. M. Millonas and D. A. Hanck, Phys. Rev. Lett. 80, 401 (1998).

[3] M. Bates, M. Burns, and A. Meller. Biophys. J. 84, 2366 (2003).

[4] O. Flomenbom and J. Klafter, Biophys. J., in press (2004).

[5] G. K. Schenter, H. P. Lu, and X. S. Xie, J. Phys. Chem. A 103, (1999).

[6] R. Zwanzig, J. Chem. Phys. 97, 3587 (1993).

[7] N. Eizenberg and J. Klafter, J. Chem. Phys. 104, 6796 (1996).

[8] C. R. Doering and J. C. Gadoua, Phys. Rev. Lett. 69, 2318 (1992).

[9] C. Van den Broeck, Phys. Rev. E. 47, 4579 (1993).

[10] U. Zürcher and C. R. Doering, Phys. Rev. E 47, 3862 (1993).

[11] M. Bier and R. D. Astumian, Phys. Rev. Lett. 71, 1649
(1993). M. Bier, I. Derényi, M. Kostur, and R. D. Astumian, Phys. Rev. E. 59, 6422 (1999).

[12] M. Boguñá, J. M. Porrà, J. Masoliver, and K. Lindenberg, Phys. Rev. E. 57, 3990 (1998).

[13] P. Reimann, R. Bartussek, and P. Hänggi, Chem. Phys. 235, (1998).

[14] O. Benichou, B. Gaveau, and M. Moreau, Phys. Rev. E. 59, 103 (1999).

[15] M. Marchi, F. Marchesoni, L. Gammaitoni, E. Menichella-Saetta, and S. Santucci, Phys. Rev. E. 54, 3479 (1996).

[16] A. Bar-Haim and J. Klafter, Phys. Rev. E. 60, 2554 (1999).

[17] R. N. Mantegna and B. Spagnolo Phys. Rev. Lett. 84, 3025 (2000).

[18] R. Zwanzig Nonequilibrium Statistical Mechanics. Oxford University Press, NY, NY, 2001.

[19] B. Alberts, K. Roberts, D. Bray, J. Lewis, M. Raff, and J. D. Watson. Molecular Biology of The Cell. Garland Publishing, Inc, NY \& London, 1994. 